\title{
Enhanced Bone Regeneration of Cortical Segmental Bone Defects Using Porous Titanium Scaffolds Incorporated with Colloidal Gelatin Gels for Time- and Dose-Controlled Delivery of Dual Growth Factors
}

\author{
Johan van der Stok, MD, ${ }^{1, *}$ Huanan Wang, MSc, ${ }^{2, *}$ Saber Amin Yavari, MSc, ${ }^{3}$ Michiel Siebelt, MD, \\ Marjan Sandker, MD, ${ }^{1}$ Jan H. Waarsing, PhD, ${ }^{1}$ Jan A.N. Verhaar, MD, PhD, ${ }^{1}$ Holger Jahr, PhD, \\ Amir A. Zadpoor, $\mathrm{PhD}{ }^{3}$ Sander C.G. Leeuwenburgh, $\mathrm{PhD}{ }^{2}$ and Harrie Weinans, $\mathrm{PhD}^{1,3,4}$
}

Porous titanium scaffolds are a promising class of biomaterials for grafting large bone defects, because titanium provides sufficient mechanical support, whereas its porous structure allows bone ingrowth resulting in good osseointegration. To reinforce porous titanium scaffolds with biological cues that enhance and continue bone regeneration, scaffolds can be incorporated with bioactive gels for time- and dose-controlled delivery of multiple growth factors (GFs). In this study, critical femoral bone defects in rats were grafted with porous titanium scaffolds incorporated with nanostructured colloidal gelatin gels. Gels were loaded with bone morphogenetic protein-2 (BMP-2, $3 \mu \mathrm{g}$ ), fibroblast growth factor-2 (FGF-2, $0.6 \mu \mathrm{g}$ ), BMP-2, and FGF-2 (BMP-2/FGF-2, ratio 5:1) or were left unloaded. GF delivery was controlled by fine tuning the crosslinking density of oppositely charged nanospheres. Grafted femurs were evaluated using in vivo and ex vivo micro-CT, histology, and three-point bending tests. All porous titanium scaffolds containing GF-loaded gels accelerated and enhanced bone regeneration: BMP-2 gels gave an early increase ( $0-4$ weeks), and FGF-2 gels gave a late increase (8-12 weeks). Interestingly, stimulatory effects of $0.6 \mu \mathrm{g}$ FGF-2 were similar to a fivefold higher dose of BMP-2 $(3 \mu \mathrm{g})$. BMP-2/FGF-2 gels gave more bone outside the porous titanium scaffolds than gels with only BMP-2 or FGF-2, resulted in bridging of most defects and showed superior bone-implant integrity in three-point bending tests. In conclusion, incorporation of nanostructured colloidal gelatin gels capable of time- and dose-controlled delivery of BMP-2 and FGF-2 in porous titanium scaffolds is a promising strategy to enhance and continue bone regeneration of large bone defects.

\section{Introduction}

$\mathbf{R}$ EGENERATION OF LARGE BONE DEFECTS remains a challenging topic in trauma, orthopedic, and maxillofacial surgery. ${ }^{1}$ Despite availability of several biomaterials for bone grafting, ${ }^{2}$ autologous bone remains the clinical gold standard. ${ }^{3}$ Nevertheless, well-known drawbacks (e.g., limited amounts and donor-site morbidity ${ }^{4}$ ) provide continuous incentive to develop new biomaterials. A promising new biomaterial for large bone defects is porous titanium. Porous titanium scaffolds directly provide mechanical support after implantation, whereas the porous structure acts as an osteoconductive $^{5}$ scaffold that facilitates bone regeneration. ${ }^{6}$ However, to further enhance and continue bone regeneration in large bone defects, it is necessary to reinforce the bioinert titanium with biological cues stimulating bone regeneration.

Bone regeneration is a cascade of events strongly regulated by spatial and temporal presentation of angiogenic and osteogenic growth factors (GFs). ${ }^{7}$ Angiogenic GFs such as fibroblast growth factor-2 (FGF-2) are expressed during early stages of bone regeneration. ${ }^{7,8}$ In contrast, osteogenic GFs such as bone morphogenetic factor-2 (BMP-2) are expressed in later stages. ${ }^{9}$ Mimicking this spatiotemporal presentation of GFs by controlled delivery from carrier materials is

Abstract was presented at the 2013 Orthopaedic Research Society annual meeting (San Antonio, US) and the 2012 European Orthopaedic Research Society annual meeting 2012 (Amsterdam, The Netherlands).

${ }^{1}$ Orthopaedic Research Laboratory, Department of Orthopaedics, Erasmus University Rotterdam Medical Centre, Rotterdam, The Netherlands.

${ }^{2}$ Department of Biomaterials, Radboud University Nijmegen Medical Centre, Nijmegen, The Netherlands.

${ }^{3}$ Department of Biomechanical Engineering, Delft University of Technology, Delft, The Netherlands

${ }^{4}$ Department of Orthopaedics and Rheumatology, Utrecht University Medical Center, Utrecht, The Netherlands.

*These authors contributed equally to this work. 
considered a powerful tool to stimulate bone regeneration. ${ }^{10}$ Conventional carrier materials (e.g., collagen sponges) have shown poor capacity to control GF delivery, and therefore require a high dose of GFs to reach therapeutic effects. However, high doses of GF, especially BMP-2, can cause serious side effects, including bone tissue overgrowth, ectopic bone formation, inflammation, and even carcinogenicity. ${ }^{11}$ This underlines the need for new carrier materials capable of time- and dose-controlled delivery of multiple GFs. ${ }^{9}$

To this end, a new generation of carrier materials recently emerged are shear-thinning and self-healing colloidal gels. ${ }^{12,13}$ Nanostructured colloidal gels made of oppositely charged gelatin nanospheres can control GF delivery by tailoring the degradation rate of the subpopulation of charged gelatin nanospheres within the gel network. It has been shown that by separately loading FGF-2 and BMP-2 using different species of charged gelatin nanospheres and fine-tuning the crosslinking densities of gelatin particles, a sequential release of dual GFs, in terms of early delivery of FGF-2 and sustained delivery of BMP-2, can be obtained. ${ }^{14}$ However, due to the inappropriate dose combination between FGF-2 and BMP-2, the dual GF delivery resulted into an inhibitory effect on bone regeneration. ${ }^{15,16}$

The aim of this study is to enhance bone regeneration of large bone defects using porous titanium scaffolds incorporated with nanostructured colloidal gelatin gels for time- and dose-controlled delivery of a nanogram dose of FGF-2 and/ or a microgram dose of BMP-2. We hypothesize that grafting cortical femoral bone defects in rats with porous titanium scaffolds containing BMP-2/FGF-2 gels result in more bone regeneration than scaffolds containing BMP-2 gels, FGF-2 gels, or unloaded gels. To test this hypothesis, bone regeneration was evaluated using in vivo and ex vivo micro-CT, histology, and three-point bending tests.

\section{Materials and Methods}

\section{Materials}

Gelatin A (GelA, from porcine skin, 300 Bloom, isoelectric point (IEP) 9) and gelatin B (GelB, from bovine skin, 225 Bloom, IEP 5) were purchased from Sigma-Aldrich. Glutaraldehyde (GA, $25 \mathrm{wt} \%$ solution in water) was commercially available from Acros Organics. Recombinant human BMP-2 (molecular mass $26 \mathrm{kDa}$ (dimer), IEP 7) was purchased from Shanghai Rebone Biomaterials Co., China, and recombinant human FGF-2 (molecular mass 16 kDa, IEP 9.6) was supplied by R\&D Systems.

\section{Preparation and characterization of GF-loaded colloidal gelatin gels}

Colloidal gelatin gels were made of oppositely charged gelatin nanospheres. ${ }^{17}$ Briefly, gelatin nanospheres were obtained using a desolvation method and crosslinked using GA. A crosslinking density (defined as molar ratios of GA relative to $\left[\mathrm{NH}_{2}\right]_{\text {gelatin }}$ ) of one (low) or four (high) was applied to GelA and GelB nanospheres, respectively. That resulted in positively charged GelA and negatively charged GelB nanospheres. Particles size and $\zeta$-potential of gelatin nanospheres were measured using dynamic light scattering (DLS, Zetasizer Nano-Z; Malvern Instruments Ltd.)
Table 1. Characteristics of Gela and GelB Nanospheres (NS)

\begin{tabular}{lcc}
\hline Characteristics & GelA NS & GelB NS \\
\hline $\begin{array}{l}\text { Crosslinking } \\
\text { density }\end{array}$ & Low $\left(\mathrm{GA} / \mathrm{NH}_{2}=1\right)$ & High $\left(\mathrm{GA} / \mathrm{NH}_{2}=4\right)$ \\
$\begin{array}{l}\text { Particle size } \\
\text { (swollen state) }\end{array}$ & $430 \pm 4 \mathrm{~nm}$ & $247 \pm 2 \mathrm{~nm}$ \\
\begin{tabular}{l} 
Zeta-potential \\
\hline
\end{tabular} & $+9.3 \pm 0.3 \mathrm{mV}$ & $-20.0 \pm 0.4 \mathrm{mV}$ \\
\hline
\end{tabular}

(Table 1). Lyophilized GelA or GelB nanospheres were mixed with aqueous $1 \mathrm{mM} \mathrm{NaCl}$ solutions ( $\mathrm{pH} 7$ ) in two separate $1-\mathrm{mL} \mathrm{BD}^{\circledR}$ Luer-lok ${ }^{\mathrm{TM}}$ syringes. A homogeneous gel was obtained by repeated extrusion from both syringes while being connected by a Luer-lok connector. BMP-2/FGF-2 gels were made mixing GelA nanospheres with a $\mathrm{NaCl}$ solution containing $22 \mu \mathrm{g} / \mathrm{mL}$ FGF-2 and mixing GelB nanospheres with a $\mathrm{NaCl}$ solution containing $110 \mu \mathrm{g} / \mathrm{mL}$ BMP-2. BMP-2, FGF-2, or unloaded gels were obtained by mixing GelA, GelB, or both with a $1 \mathrm{mM} \mathrm{NaCl}$ solution only (Table 2). Gels (solid content of $20 \mathrm{w} / \mathrm{v} \%$ ) were stored at $4^{\circ} \mathrm{C}$ overnight to allow for complete swelling and GF absorption. Viscoelastic properties were characterized using a rheometer (AR2000ex; TA Instruments). Measurements to assess shear-thinning and self-healing behavior were performed within the linear viscoelastic region using a flat steel plate geometry $(20 \mathrm{~mm}$ diameter $)$ at $25^{\circ} \mathrm{C}$. Briefly, gels were gradually destroyed by applying an oscillatory strain sweep with shear strain increasing from $0.1 \%$ to $1000 \%$ ( $1 \mathrm{~Hz}$ frequency); meanwhile, viscoelastic properties $\left(G^{\prime}\right.$ and $\left.G^{\prime \prime}\right)$ were monitored. Thereafter, an oscillatory time sweep ( $5 \mathrm{~min}, 1 \%$ strain, $1 \mathrm{~Hz}$ frequency) was instantaneously applied and gel recovery was recorded.

\section{Incorporation of GF-loaded colloidal gelatin gels into porous titanium scaffolds}

The selective laser melting (SLM) technique was used to produce porous titanium scaffolds in the shape of the critical femoral bone segment removed during the animal experiment (Fig. 1A). ${ }^{6}$ The porous structure was based on a dodecahedron template designed with $120 \mu \mathrm{m}$ thick titanium struts and pores ranging from 240 to $730 \mu \mathrm{m}$. Porosity, compression strength, and Young's modulus are given in Table 3. Postproduction, scaffolds underwent an alkali-acidheat treatment as described previously. ${ }^{18}$

Gels were incorporated into the scaffolds using a custommade gel chamber before surgery. First, a scaffold was placed inside the gel chamber and subsequently, the gel chamber was connected to the Luer-lok syringes that contained the gels. Then, an excess of gel was injected into the

\begin{tabular}{lcc} 
& $\begin{array}{c}\text { Table 2. Preparation of (GF-loaded) } \\
\text { Colloidal Gelatin Gels }\end{array}$ \\
\hline Gels & GelA & GelB \\
\hline Unloaded & - & - \\
FGF-2 & $22 \mu \mathrm{g} / \mathrm{mL}$ FGF-2 & - \\
BMP-2 & - & $110 \mu \mathrm{g} / \mathrm{mL} \mathrm{BMP-2}$ \\
BMP-2/FGF-2 & $22 \mu \mathrm{g} / \mathrm{mL}$ FGF-2 & $110 \mu \mathrm{g} / \mathrm{mL} \mathrm{BMP-2}$ \\
\hline
\end{tabular}

FGF-2, fibroblast growth factor-2; BMP-2, bone morphogenetic factor-2. 


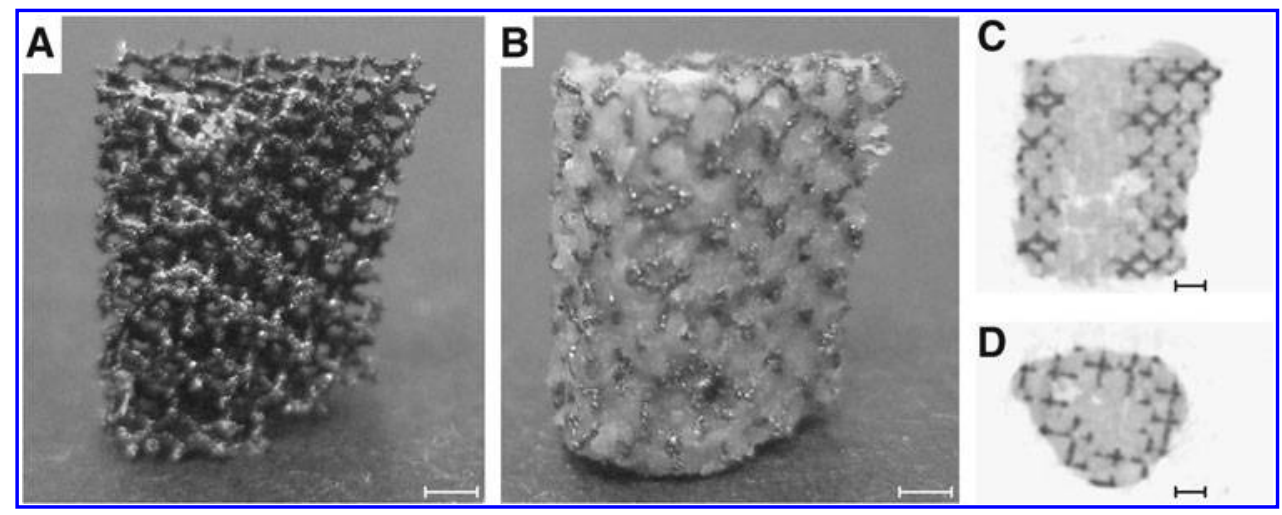

FIG. 1. Photographs of porous titanium scaffolds in the shape of the 6-mm bone segment that was replaced during the animal experiment before (A) and after (B) incorporation with colloidal gelatin gels. Micro-CT images of perpendicular (C) and horizontal (D) cross sections of porous titanium scaffold (black) incorporated with colloidal gelatin gels containing iodine-based radiographic contrast agent ioxaglate (gray). Scale bar $=1 \mathrm{~mm}$. Micro-CT, micro-computed tomography.

chamber resulting in complete filling of the porous titanium scaffold. Gel-loaded titanium scaffolds were left inside the gel chamber and stored at $4^{\circ} \mathrm{C}$ until implantation at which, superfluous gel around the scaffold was removed (Fig. 1B). Filling efficacy of the porous space was visualized using micro-CT and scanning electron microscopy (SEM). Therefore, the gel was prepared with a contrast agent (Hexabrix $320^{\circledR}$; Mallinckrodt), subsequently scanned with a SkyScan 1176 micro-CT scanner (Bruker micro-CT N.V). Complete filling of the porous space $\left(55 \mathrm{~mm}^{3}\right)$ resulted in a total dose of $3 \mu \mathrm{g} \mathrm{BMP-2}$ and/or 0.6 $\mu \mathrm{g}$ FGF-2.

\section{Animal experiment}

In 40 male Wistar rats, a critical femoral bone defect was grafted with porous titanium scaffolds loaded with the four different colloidal gelatin gels (Table 2). The Animal Ethics Committee of the Erasmus University approved the study and Dutch guidelines for care and use of laboratory animals were applied. Before surgery, rats received a single dose of antibiotics (enrofloxacin, $5 \mathrm{mg} / \mathrm{kg}$ body weight). Surgery was performed aseptically under general anesthesia (1-3.5\% isoflurane). The right femur was exposed through a lateral skin incision and division of underlying fascia. A $23 \mathrm{~mm}$ long PEEK plate was fixated to the femur anterolateral plane using three proximal and three distal screws. Periosteum was removed over approximately $8 \mathrm{~mm}$ of the mid-diaphyseal region before a $6 \mathrm{~mm}$ bone segment was removed with a wire saw and a tailor-made saw guide. Subsequently, a gelloaded porous titanium scaffold was implanted press-fit into the defect. Finally, fascia and skin were sutured using Vicryl 5-0 and pain medication (buprenorphine, $0.05 \mathrm{mg} / \mathrm{kg}$ body weight) was administered twice a day for 3 days.

Table 3. Properties of Porous Titanium Scaffolds

\begin{tabular}{lr}
\hline Titanium thickness & $120 \mu \mathrm{m}$ \\
Pore size & $490 \mu \mathrm{m}$ \\
Porosity & $88 \%$ \\
Pore volume & $55 \mathrm{~mm}^{3}$ \\
Compression strength & $14 \mathrm{MPa}$ \\
Young's modulus & $0.4 \mathrm{GPa}$
\end{tabular}

\section{Micro-CT evaluation}

Immediately after surgery, a baseline in vivo micro-CT scan was acquired (SkyScan 1176 scanner; Bruker microCT N.V.) using a $35 \mu \mathrm{m}$ resolution protocol $(90 \mathrm{kV}, 0.1 \mathrm{~mm}$ $\mathrm{Cu}$ filter, and $0.5^{\circ}$ rotation step, $7 \mathrm{~min}$ scan). In vivo scans were repeated after 4, 8, and 12 weeks. At 12 weeks, an ex vivo scan was made using an $18 \mu \mathrm{m}$ resolution protocol $\left(90 \mathrm{kV}, 0.1 \mathrm{~mm} \mathrm{Cu}\right.$ filter, and $0.2^{\circ}$ rotation step, $1 \mathrm{~h}$ scan). CT images were reconstructed using volumetric reconstruction software NRecon version 1.5 (Bruker micro-CT N.V.).

Bone formation was expressed as bone volume (BV) and measured at four specific regions: (1) total BV, total volume of bone formed within the $6 \mathrm{~mm}$ defect; (2) outer BV, bone formed outside the scaffolds; (3) porous BV, bone formed inside the porous space of the scaffolds; and (4) inner BV, bone formed in the medullary canal of the scaffold. Specific BVs were measured after selecting the specific region as a volume of interest (VOI) on the scans using a custom-made algorithm within CTAnalyser software version 1.11 (Bruker micro-CT N.V.). After selection of the specific VOIs, titanium was subtracted from the images using a global threshold. Additionally, a $35 \mu \mathrm{m}$ layer of the interface was subtracted to minimize interference of metal artefacts with BV measurements. BV was then determined after applying a global threshold that was based on visual inspection and was kept constant for all scans. The extent of bone bridging was measured on ex vivo scans with DataViewer 1.4 (Bruker micro-CT N.V.).

\section{Histology}

Histology was performed on five femurs of each group to study the bone-titanium interface and bone morphology. After harvesting and removal of the surrounding soft tissues, femurs were fixed in a $10 \%$ neutral buffered formalin solution for 2 days, dehydrated in a graded ethanol solution from $70 \%$ to $100 \%$, and finally embedded in methyl methacrylate. Sections of $\sim 20 \mu \mathrm{m}$ were obtained using a diamond saw (Leica SP1600) and stained with a basic fuchsin $0.3 \%$ solution and methylene blue $1 \%$ solution. Basic fuchsin stains bone red and methylene blue stains fibrous tissue blue. 
FIG. 2. Rheological characterization of self-healing behavior of colloidal gelatin gels $(20 \mathrm{w} / \mathrm{v} \%$ solid content) by monitoring storage moduli $\mathrm{G}^{\prime}$ (square) and loss moduli G" (circle) as a function of time (region II: oscillatory time sweep with $1 \%$ strain and $1 \mathrm{~Hz}$ frequency) after network destruction by increasing strain from $0.1 \%$ to $1000 \%$ (region I: oscillatory strain sweep with $1 \mathrm{~Hz}$ frequency). Color images available online at www .liebertpub.com/tea

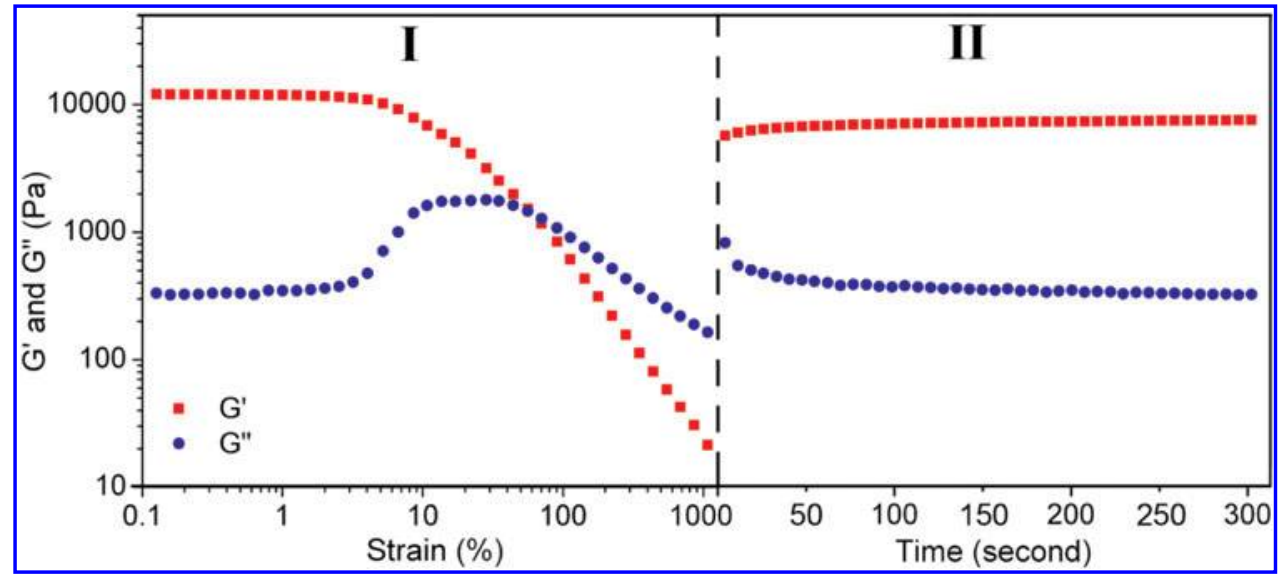

\section{Mechanical evaluation}

The final strength of five grafted femurs from each group was measured using three-point bending tests, the intact contralateral femurs served as controls. After harvesting the femurs, soft tissue and the PEEK plate were carefully removed. Specimens were kept in the $10 \%$ neutral buffered formalin solution for 2 days followed by phosphate-buffered saline, thereby minimizing the effects of formalin conservation on mechanical properties. ${ }^{19}$ Bending tests were carried out using a Zwick test machine (Zwick $\mathrm{GmbH}$ ) as follows: first, femurs were fixed at both sides using two holding plates that were secured with screws. The fixated femur was supported by two supports ( $15 \mathrm{~mm}$ width) and a downward force was applied by an indenter ( $3 \mathrm{~mm}$ width) to the middle of the anterolateral plane of the porous titanium scaffold.
Bending tests were performed at a displacement rate of $2 \mathrm{~mm} / \mathrm{min}$ until peak load was reached. Force-displacement curves were recorded and used to determine the fracture force, maximum moment, ultimate displacement and bending stiffness. Bending stiffness was determined by fitting a line to the initial linear portion of the force-displacement curve.

\section{Statistics}

Statistical analyses were performed using SPSS Statistics 20.0 (SPSS, Inc.). Data are presented as means with standard deviations. One-way analysis of variation (ANOVA) and subsequent post hoc pairwise comparisons with Bonferroni adjustment were used to analyze differences between groups.
FIG. 3. Scanning electron micrographs of the porous titanium scaffolds before (A, B) and after (C, D) incorporation of the colloidal gelatin gels. Low $(\mathbf{A}, \mathbf{C})$ and high $(\mathbf{B}, \mathbf{D})$ magnification.
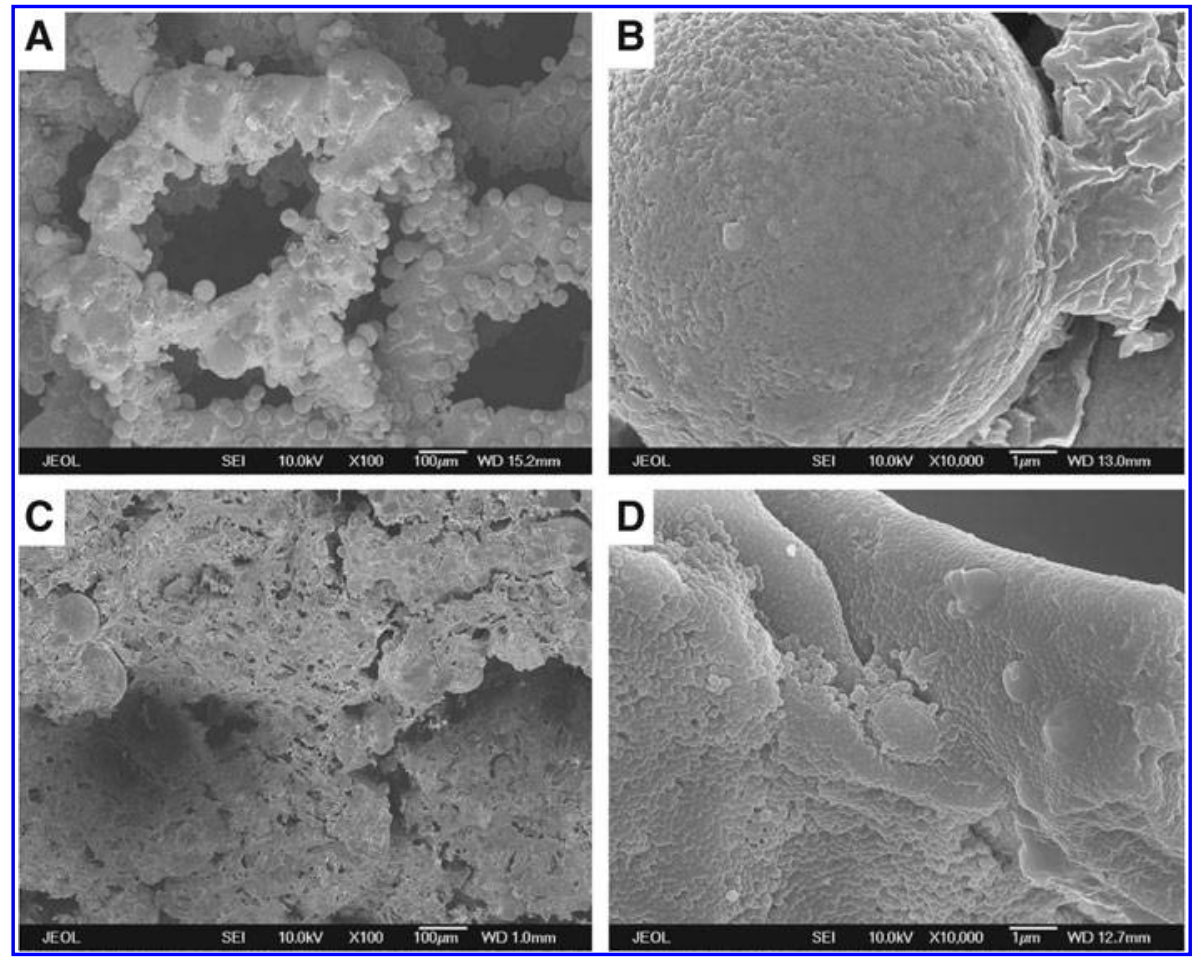


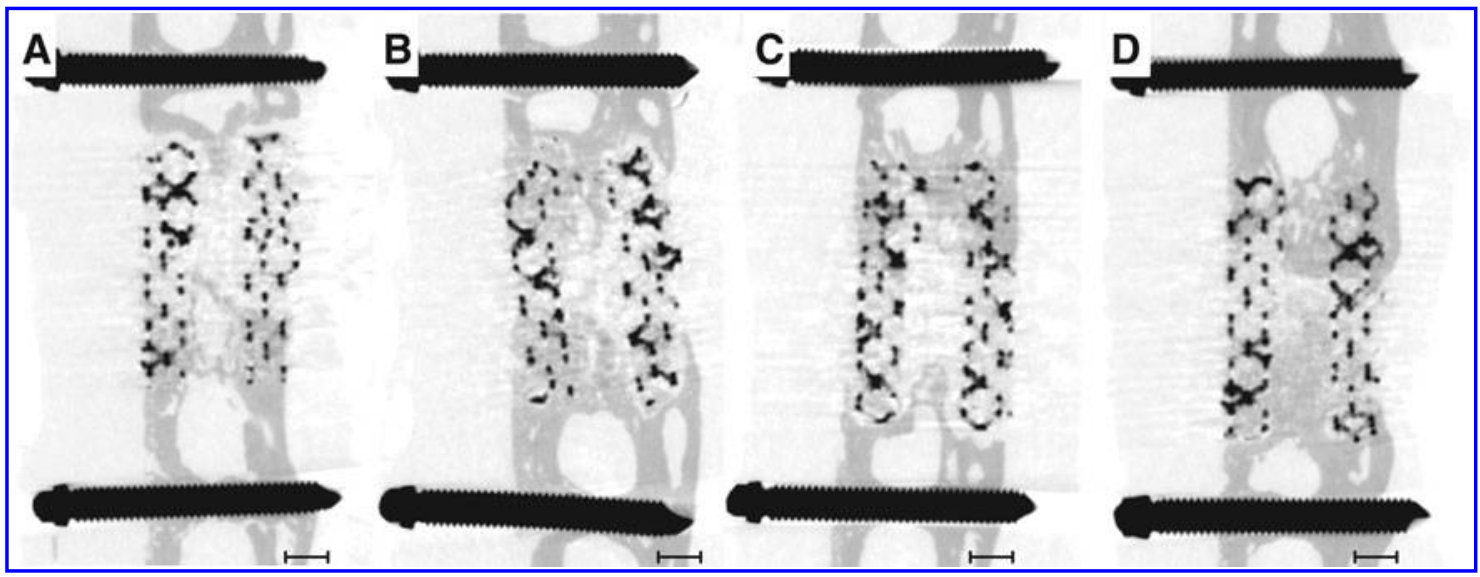

FIG. 4. Representative transversal micro-CT images of the porous titanium scaffolds containing unloaded (A), fibroblast growth factor-2 (FGF-2) (B), bone morphogenetic protein-2 (BMP-2) (C), or BMP-2/FGF-2 (D) gels after 12 weeks. Porous titanium scaffolds and fixation screws appear in black, whereas bone appears in dark gray. Scale bar $=1 \mathrm{~mm}$.

\section{Results}

\section{Gelatin nanosphere-based colloidal gels}

Rheological characterization indicates the formation of highly elastic gels, since a storage moduli $\left(\mathrm{G}^{\prime}\right)$ of $\sim 12 \mathrm{kPa}$ was substantially higher than a loss moduli $\left(\mathrm{G}^{\prime \prime}\right)$ (corresponding to tan (delta) values of about 0.03) (Fig. 2, region I). Gel networks were gradually destroyed by applying an external increasing shear strain, which led to the transformation from elastic, solid-like to liquid-like materials $\left(\mathrm{G}^{\prime}<\mathrm{G}^{\prime \prime}\right)$ at strain exceeding $70 \%$. At the removal of the external destructive shear force, it was observed that the gel strength recovered immediately, with up to $70 \%$ of the initial gel elasticity $\left(\mathrm{G}^{\prime}\right)$ recovered within 5 min (Fig. 2, region II).

Colloidal gelatin gels were easily incorporated into porous titanium scaffolds by an injection filling process using a custom-made gel chamber (Supplementary Video S1; Supplementary Data are available online at www.liebertpub .com/tea) resulting in a homogeneous distribution of the gels throughout the porous scaffolds as confirmed by micro-CT (Fig. 1C, D). SEM shows fused titanium particles forming the porous structure (Fig. 3A) and the surface morphology of titanium before (Fig. 3B) and after gel incorporation (Fig. 3C). After gel incorporation, original surface morphology of titanium was covered by nanoparticulate morphology of colloidal gelatin gels consisting of nanoparticles ranging between 100-200 $\mathrm{nm}$ in diameter (Fig. 3D), while the porous architecture of the scaffolds was covered completely by the nanostructure of a particulate gel network.

\section{Animal experiment}

Rats were able to tolerate weight-bearing activities immediately after surgery, and implantation sites healed without complications. All animals remained healthy during the entire study period, except one rat that died due to anesthesia-related complications (BMP-2 group).

\section{Micro-CT evaluation}

Correct positioning of the porous titanium scaffold, PEEKplate, and screws was confirmed directly after surgery in all animals and no dislocation was detected during follow-up.
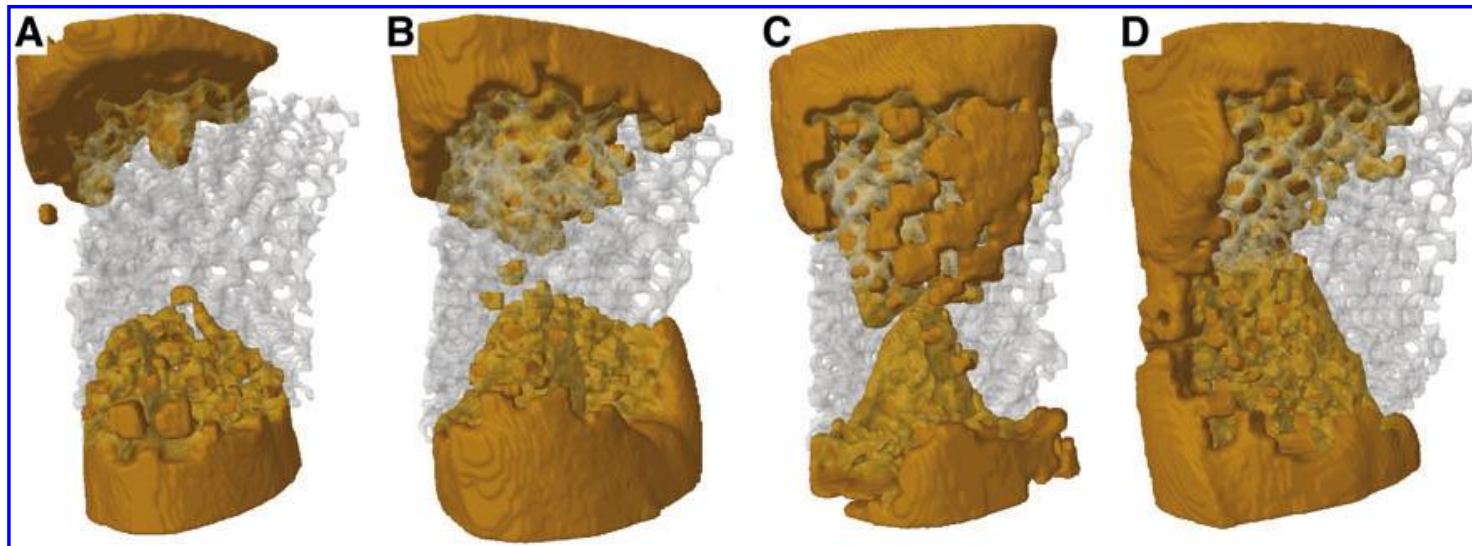

FIG. 5. Representative 3D micro-CT images of bone bridging the porous titanium scaffolds containing unloaded (A), FGF-2 (B), BMP-2 (C), or BMP-2/FGF-2 (D) gels after 12 weeks. Porous titanium scaffolds are shown in transparent, whereas bone appears in dark grey. Color images available online at www.liebertpub.com/tea 
FIG. 6. (A) In vivo micro-CT quantification of total bone volume (BV) at 4, 8, 12 weeks after implantation. (B) Ex vivo micro-CT quantification of total BV after 12 weeks. Total BV was defined as total volume of bone formed within the $6 \mathrm{~mm}$ defect area. Horizontal and vertical bars indicate statistically significant differences $(p<0.05)$ between groups.

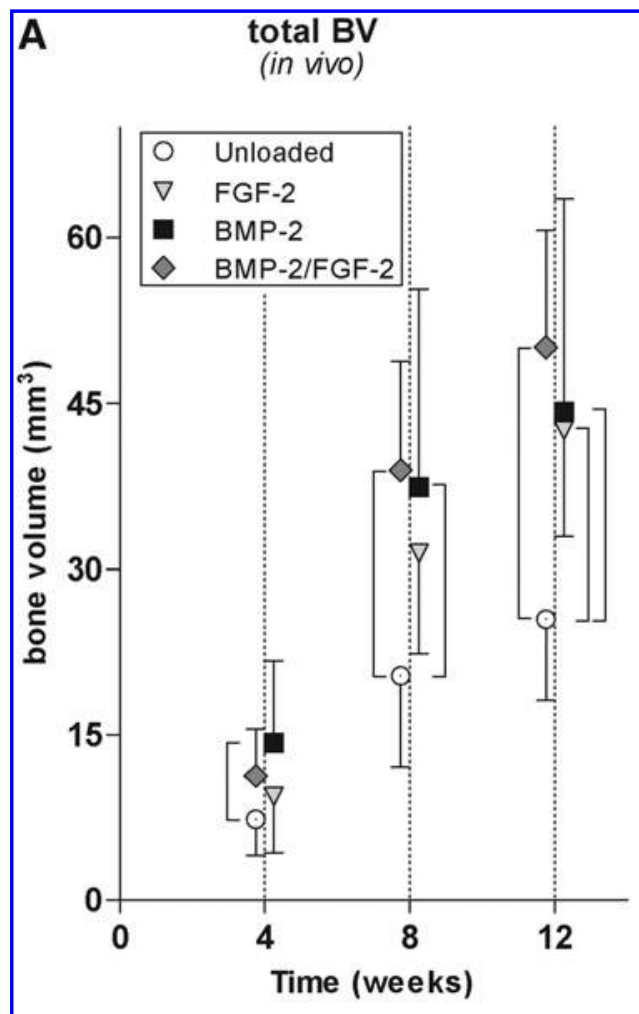

B

total BV

(ex vivo)

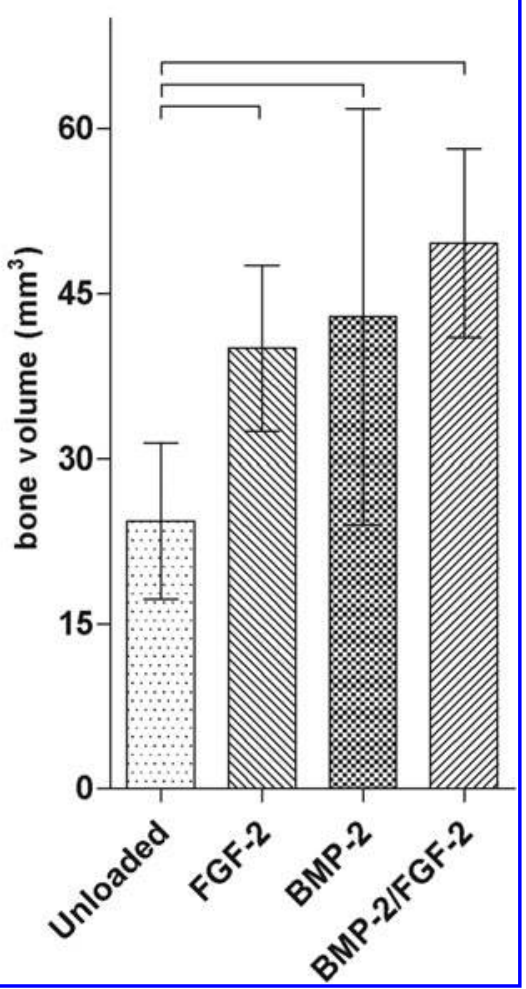

In general, bone regeneration started at the bone adjacent to the defect and progressed toward the central area of the defect (Fig. 4).

Porous titanium scaffolds almost completely bridged the defect (defined as a remaining gap $<0.5 \mathrm{~mm}$ ) in seven rats of the BMP-2/FGF-2 group, seven rats of the BMP-2 group, five rats of the FGF-2 group, and one rat of the unloaded group (Fig. 5). The average remaining gap distance was $0.33 \pm$ $0.34 \mathrm{~mm}$ for the BMP-2/FGF-2 group, $0.51 \pm 0.74 \mathrm{~mm}$ for the BMP-2 group, $0.83 \pm 0.53 \mathrm{~mm}$ for the FGF-2 group, and $1.20 \pm 0.56 \mathrm{~mm}$ for the unloaded group.
Porous titanium scaffolds with GF-loaded gels resulted in significantly more bone regeneration than porous titanium scaffolds with unloaded gels after 12 weeks (Fig. 6). BMP-2 gels strongly enhanced early bone formation, since total BV was significantly more than with unloaded gels at 4 weeks (Fig. 6A). Total BV continued to increase between 4 and 8 weeks, and reached a plateau phase after 8 weeks. FGF-2 gels enhanced bone formation in the later phase, as total BV increased rapidly between 4 and 8 weeks. Finally, FGF-2 gels resulted in $40.0 \pm 7.6 \mathrm{~mm}^{3}$ total $\mathrm{BV}$, which was similar to BMP-2
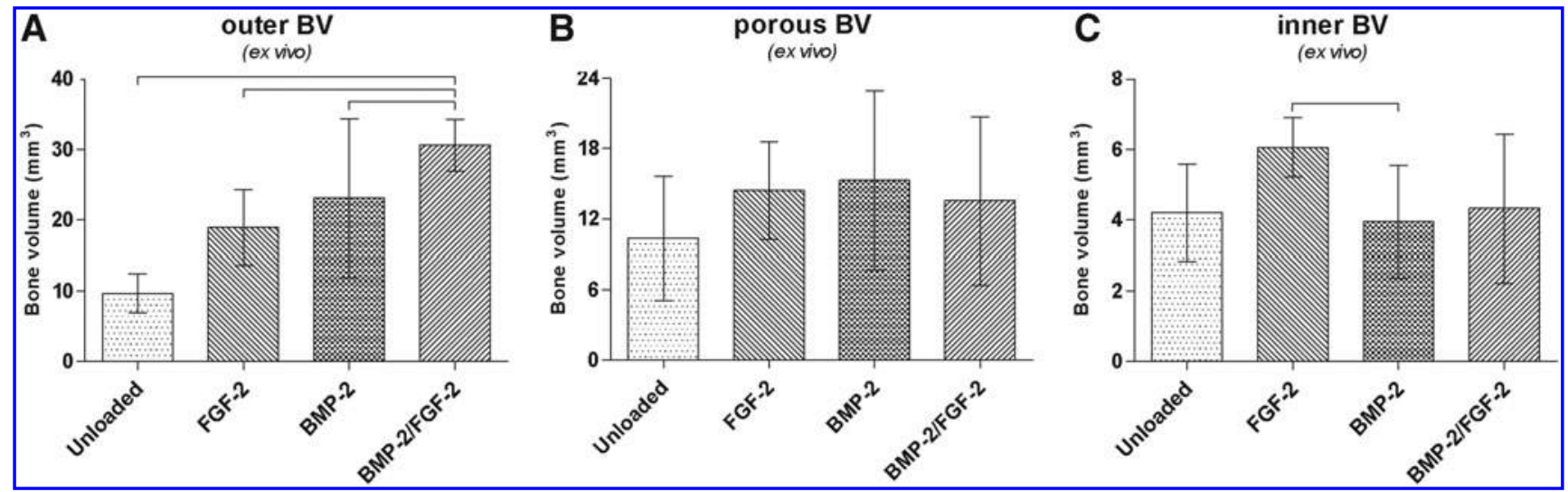

FIG. 7. Ex vivo micro-CT quantification of outer BV (A), porous BV (B), and inner BV (C) at 12 weeks. Outer BV was defined as bone formed outside the porous titanium scaffolds; porous BV, bone formed inside the porous space of the titanium scaffolds; and inner BV, bone formed in the medullary canal of the scaffold. Horizontal bars indicate statistical significant differences $(p<0.05)$. 


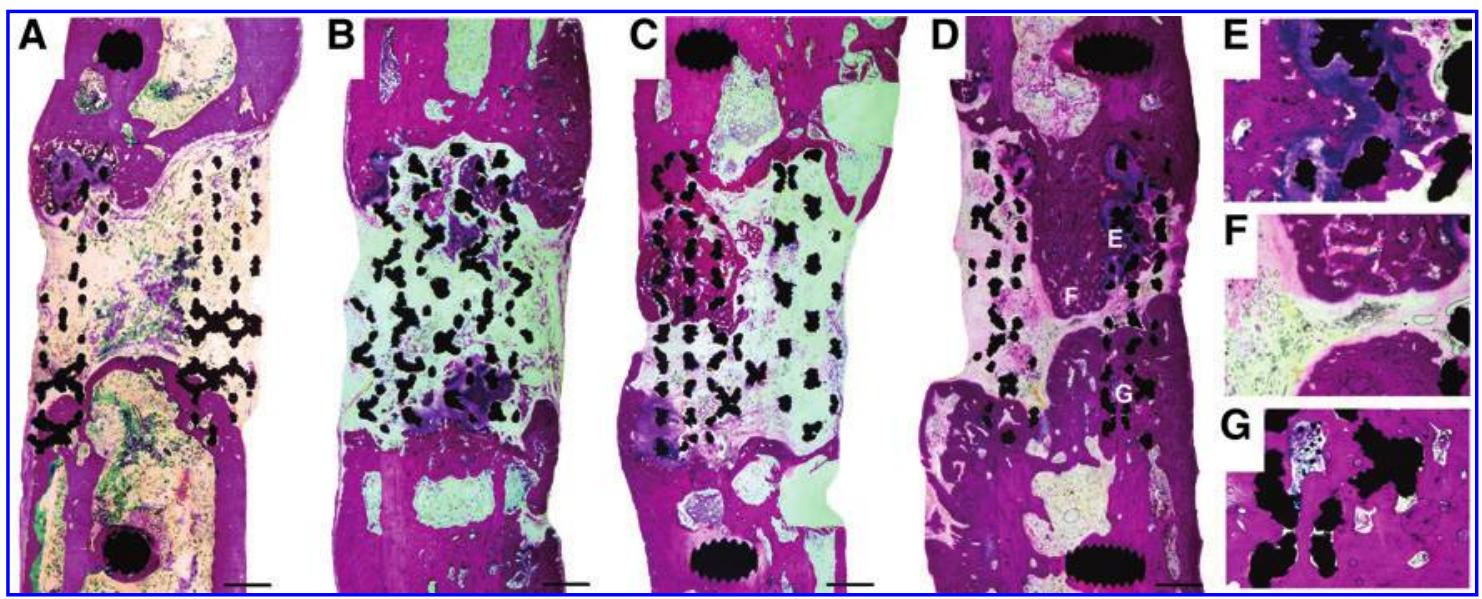

FIG. 8. Representative transversal histological sections of the porous titanium scaffolds containing unloaded (A), FGF-2 (B), BMP2 (C), or BMP-2/FGF-2 (D) gels after 12 weeks as well as high-magnification views (E-G) of the areas as depicted in (D). Titanium appears in black, whereas bone appears in dark grey. Scale bar $=1 \mathrm{~mm}$. Color images available online at www.liebertpub.com/tea

gels $\left(42.9 \pm 18.9 \mathrm{~mm}^{3}\right)$. BMP-2/FGF-2 gels continuously enhanced bone formation during the entire follow-up period, resulting in the highest average total $\mathrm{BV}$ at 12 weeks $\left(49.6 \pm 8.6 \mathrm{~mm}^{3}\right)$ (Fig. 6B).

Porous titanium scaffolds with BMP-2/FGF-2 gels resulted in significantly more bone outside the porous titanium scaffolds (outer BV) than those with BMP-2 gels, or FGF-2 gels (Fig. 7A). Bone ingrowth into the porous titanium scaffold (porous BV), however, was not significantly enhanced by GF-loaded gels (Fig. 7B). FGF-2 gels resulted in significantly more bone inside the porous titanium scaffold (inner BV) than BMP-2 gels $\left[6.1 \pm 0.8 \mathrm{~mm}^{3}\right.$ versus $4.0 \pm 1.6 \mathrm{~mm}^{3}(p=0.033)$, Fig. 7C].

\section{Histological evaluation}

No distinct differences in terms of bone morphology, bone-titanium bonding, or vascularization between experimental groups were observed (Fig. 8A-D). Areas of newly formed bone differed considerably, unloaded gels showed less bone (Fig. 8A) than FGF-2 gels (Fig. 8B), BMP-2 gels (Fig. 8C), and BMP-2/FGF-2 gels (Fig. 8D). Bone formation inside the porous space was mainly located at distal and proximal sites and resulted in direct bone-titanium contact (Fig. 8G). A direct bone-titanium contact was not found throughout the entire bone-titanium interface. In some areas, a cartilage-like zone was observed (Fig. 8E). Fibrous tissue infiltration was occasionally seen inside pores devoid of newly formed bone and within the remaining gap of nearly completely bridged defects (Fig. 8F). No remnants of colloidal gelatin gels were detected, and no signs of foreign body reaction or inflammatory response were observed.

\section{Mechanical evaluation}

All grafted femurs broke at approximately 50\% of the force required to break the intact contralateral femurs, but fracture force and ultimate displacement did not show statistically significant differences between the groups (Table 4). The observed fracture location, however, differed substantially. Femurs grafted with scaffolds containing unloaded gels broke at the bone-implant interface $(2 / 5)$ or through the porous titanium scaffolds (3/5). Similar fracture locations were observed for scaffolds containing FGF-2 gels. In contrast, the BMP-2 group consistently broke at the bone-implant interface (4/4) and the BMP-2/FGF-2 group fractured consistently (5/5) through the host bone at $\sim 3 \mathrm{~mm}$ distally from the titanium-bone interface.

\section{Discussion}

Porous titanium scaffolds have recently received increasing attention as a new biomaterial that can act as an osteoconductive scaffold that provides direct mechanical support in large bone defects at weight-bearing sites. ${ }^{20}$ Advanced additive manufacturing methods, such as SLM,

Table 4. Fracture Locations of the Three-Point Bending Tests

\begin{tabular}{|c|c|c|c|c|c|c|c|}
\hline \multirow[b]{2}{*}{ Gels } & \multirow[b]{2}{*}{$\begin{array}{l}\text { Fracture } \\
\text { force }(N)\end{array}$} & \multirow[b]{2}{*}{$\begin{array}{c}\text { Ultimate } \\
\text { displacement }(\mathrm{mm})\end{array}$} & \multicolumn{3}{|c|}{ Fracture location } & \multirow[b]{2}{*}{$\begin{array}{c}\text { Bending } \\
\text { stiffness }(\mathrm{N} / \mathrm{mm})\end{array}$} & \multirow[b]{2}{*}{$\begin{array}{c}\text { Maximum } \\
\text { moment (N.mm) }\end{array}$} \\
\hline & & & $\begin{array}{l}\text { Host } \\
\text { bone }\end{array}$ & $\begin{array}{c}\text { Bone-implant } \\
\text { interface }\end{array}$ & Implant & & \\
\hline Unloaded & $119 \pm 71$ & $2.5 \pm 0.9$ & & $2 / 5$ & $3 / 5$ & $104 \pm 19$ & $50 \pm 30$ \\
\hline FGF-2 & $122 \pm 72$ & $1.9 \pm 0.7$ & & $3 / 5$ & $2 / 5$ & $105 \pm 21$ & $51 \pm 30$ \\
\hline BMP-2 & $157 \pm 69$ & $2.7 \pm 0.9$ & & $4 / 4$ & & $109 \pm 52$ & $66 \pm 29$ \\
\hline BMP-2/FGF-2 & $111 \pm 38$ & $2.9 \pm 1.0$ & $5 / 5$ & & & $100 \pm 28$ & $47 \pm 16$ \\
\hline Intact femurs & $254 \pm 55$ & $0.9 \pm 0.3$ & & & & $428 \pm 161$ & $107 \pm 24$ \\
\hline
\end{tabular}


allow precise and reproducible production of porous titanium with full control over the structural design. Thereby, the structural design can now be fine-tuned so that porous titanium scaffolds offer optimal mechanical and structural conditions required for bone regeneration. ${ }^{6}$ However, porous titanium scaffolds remain bioinert and thereby it lacks the ability to provide strong biological cues that can enhance bone regeneration. This lead to the hypothesis that when GF-loaded colloidal gelatin gels are incorporated into porous titanium scaffolds this would result in a biologically active and mechanically strong composite biomaterial that can enhance bone regeneration of large bone defects.

Incorporation of colloidal gelatin gels was easily obtained (Fig. 1C, D) owing to shear-thinning and self-healing capacity of the gels, shown by fast recovery from severe network destruction during rheological testing (Fig. 2). Moreover, the gels used were previously shown to exhibit a strong capacity for controlled and sustained delivery of multiple GFs by employing different subpopulations of gelatin nanospheres of different crosslinking densities. Crosslinking densities of gelatin nanospheres resulted in a rapid delivery of FGF-2 and a sustained delivery of BMP-2, whereas the gelatin gel is resorbed in 4 weeks. ${ }^{14}$

Generally, this in vivo study confirms the load-bearing and osteoconductive properties of porous titanium scaffolds, as well as the biological efficacy of GF delivery by nanostructured colloidal gelatin gels. The presence of colloidal gels inside the porous space during the first 4 weeks did not seem to hamper bone regeneration, because total BVs measured in defects grafted with porous titanium scaffolds that contained unloaded gels were similar to total BVs measured in empty porous titanium scaffolds that were used in our previous study. ${ }^{6}$ Although direct bone-titanium contact was observed in all groups, occasionally, cartilage-like tissue was formed at parts of the interface (Fig. 8E). This cartilage-like tissue may indicate that micromotion had been possible at some areas between the titanium scaffold and surrounding tissues. Although bone healing generally benefits from a certain degree of micromotion, excessive micromotion is known to inhibit bone calcification at the fracture union site. ${ }^{21}$ This could also explain the fact that, although grafting with titanium scaffolds containing BMP-2/FGF-2 gels or BMP-2 gels resulted in almost complete bridging, none was fully bridged (Fig. 8F). Inability to bridge the defect can likely be overcome by using a more rigid plate.

Incorporation of BMP-2/FGF-2 gels resulted in significantly more bone after 12 weeks than incorporation of unloaded gels. However, no significant differences in total BV were observed between different GF-loaded gels. Based on previous studies related to BMP-2 delivery in $6 \mathrm{~mm}$ segmental bone defects in rat femurs, a BMP-2 dosage of $3 \mu \mathrm{g}$ per defect was considered safe and efficient in stimulating osteogenesis. $^{22,23}$ The stimulatory effect of BMP-2 gels became apparent during the early stages of bone healing (0-8 weeks), as reflected by significantly more bone at 4 and 8 weeks compared to unloaded gels (Fig. 6A). This effect was also observed in a previous in vivo study, where BMP-2 gels in rat femoral condyle defects led to significant enhancement of bone formation. ${ }^{14}$ Bone regeneration slowed down after 8 weeks, which may be explained by complete depletion of the pool of progenitor cells in and around the bone defect site that are capable of differentiating into osteoblasts upon BMP2 stimulation.

On the other hand, rapid delivery of $0.6 \mu \mathrm{g}$ FGF-2 per defect resulted in comparable amounts of bone formed after 12 weeks as the sustained delivery of a five times higher doses of BMP-2. This stresses the potent stimulatory effects of low-dose FGF-2 (nanograms) on bone regeneration in vivo. ${ }^{24}$ Although FGF-2 is expected to be delivered rapidly from colloidal gelatin gels used, ${ }^{14}$ stimulatory effects were more profound during later stages of bone healing (4-12 weeks) (Fig. 6A). It was not possible to elucidate the exact mechanism of action of FGF-2 in this study, but since FGF-2 stimulates proliferation of progenitor cells, it can be speculated that FGF-2 contributed to an expanded pool of osteoprogenitor cells that subsequently can undergo osteogenic differentiation resulting in more bone formation in a later phase. $^{24,25}$ In addition, angiogenic effects of FGF-2 can potentially lead to enhanced vascularization, which may become crucial in continuation of bone regeneration after 4 weeks. ${ }^{26,27}$ Finally, despite the absence of significant differences in total BV at 12 weeks between BMP-2 gels and FGF-2 gels, significantly more bone was observed within the medullary canal (inner BV) with FGF-2 gels (Fig. 7C) suggesting that the proliferative effect of FGF-2 acts stronger on endosteal rather than periosteal cells. ${ }^{28}$

BMP-2/FGF-2 gels incorporated into titanium scaffolds resulted in a continuous increase in total BV. Total BV after 12 weeks was not statistically different compared to BMP-2 gels or FGF-2 gels, but significantly more bone was formed outside the porous titanium scaffolds. This could be explained by the higher availability and/or more efficient attraction of osteoprogenitor cells from surrounding tissues at the outer side of titanium scaffolds compared to the inner region. More interesting is the fact that in a previous study, colloidal gelatin gels loaded with a 1:1 dose ratio of BMP-2 and FGF-2 ( $2 \mu \mathrm{g}$ per defect) had inhibitory effects on in vivo bone regeneration. ${ }^{14}$ This inhibitory effect was likely caused by overdosing FGF-2 relative to BMP-2. Similar observations on combined delivery of BMP-2 and FGF-2 indicate that higher doses of FGF-2 (micrograms) with lower or equal doses of BMP-2 generally hampered bone formation. ${ }^{15,29,30}$ In line with our results, using a 5:1 dose ratio of BMP-2:FGF2, a lower dose of FGF-2 (nanograms) in combination with higher doses of BMP-2 (micrograms) is found to stimulate osteogenesis. $^{31,32}$

Mechanical strength of grafted femurs was assessed using three-point bending tests, which aimed to evaluate the strength of the interface between porous titanium scaffolds and adjacent host bone. The absence of significant differences in maximum force suggests that only a limited amount of bone ingrowth is effective for complete biomechanical integration of porous titanium scaffolds with adjacent host bone (Table 4). The compression strength of porous titanium scaffolds used here is $14 \mathrm{MPa}$ (Table 2). This low strength can explain the fractures that occurred within the titanium scaffolds or at the bone-implant interface when the applied force of the indenter $(3 \mathrm{~mm}$ width) exceeded $100 \mathrm{~N}$. The fracture location of femurs grafted with porous titanium scaffolds containing BMP-2/FGF-2 gels is particularly interesting because BMP-2/FGF-2 gels consistently shifted the weakest link from the bone-implant interface toward the host bone at point $\sim 3 \mathrm{~mm}$ distally of the scaffold. This observation 
suggested that the extensive bone formed outside the porous titanium scaffolds incorporated with BMP-2/FGF-2 gels could further strengthen the mechanical properties of the scaffolds. Overall, the fact that grafted femurs could already sustain bending forces up to $50 \%$ of that required to break an intact femur after 12 weeks, shows the potential of porous titanium scaffolds for grafting large weight-bearing bone defects.

\section{Conclusions}

In the current study, porous titanium scaffolds were reinforced with nanostructured colloidal gelatin gels loaded with BMP-2 and/or FGF-2 to graft critical femoral bone defect in rats. Results clearly demonstrated stimulatory effects of time- and dose-controlled delivery of BMP-2, FGF-2, or both on bone regeneration. Most defects grafted with porous titanium scaffolds containing BMP-2/FGF-2 gels or BMP-2 gels were almost completely bridged after 12 weeks. BMP-2/ FGF-2 gels resulted in significantly more bone outside the porous titanium scaffolds. The stimulatory effect of delivery of merely $0.6 \mu \mathrm{g}$ FGF-2 per defect was similar to delivery of a

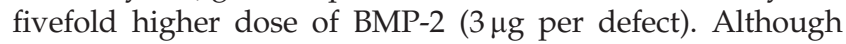
three-point bending tests did not show significant differences in fracture force, the pattern of fracture locations indicated that BMP-2/FGF-2 gels enhanced the titanium-bone interface strength thereby shifting the weakest point from the titanium-bone interface toward the surrounding femoral bone. In summary, incorporation of nanostructured colloidal gelatin gels capable of time- and dose-controlled delivery of FGF-2 and BMP-2 into porous titanium scaffolds offers a mechanically strong and biologically active composite biomaterial suitable for grafting large bone defects.

\section{Acknowledgments}

This research forms part of the Project P2.04 BONE-IP of the research program of the BioMedical Materials institute, cofunded by the Dutch Ministry of Economic Affairs. We are also grateful for the support from funding KNAW, ChinaNetherlands Programme Strategic Alliances (PSA) (No.2008DFB50120).

\section{Disclosure Statement}

No competing financial interests exist.

\section{References}

1. Langer, R., and Vacanti, J.P. Tissue engineering. Science 260, 920, 1993.

2. Van der Stok, J., Van Lieshout, E.M.M., El-Massoudi, Y., Van Kralingen, G.H., and Patka, P. Bone substitutes in the Netherlands - a systematic literature review. Acta Biomater 7, 739, 2011.

3. Cypher, T.J., and Grossman, J.P. Biological principles of bone graft healing. J Foot Ankle Surg 35, 413, 1996.

4. Banwart, J.C., Asher, M.A., and Hassanein, R.S. Iliac crest bone graft harvest donor site morbidity. A statistical evaluation. Spine 20, 1055, 1995.

5. Albrektsson, T., and Johansson, C. Osteoinduction, osteoconduction and osseointegration. Eur Spine J 10 Suppl 2, S96, 2001.
6. Van der Stok, J., Van der Jagt, O.P., Amin Yavari, S., De Haas, M.F.P., Waarsing, J.H., Jahr, H., et al. Selective laser melting produced porous titanium scaffolds regenerate bone in critical size cortical bone defects J Orthop Res 31, 792, 2013.

7. Schmid, G.J., Kobayashi, C., Sandell, L.J., and Ornitz, D.M. Fibroblast growth factor expression during skeletal fracture healing in mice. Dev Dyn 238, 766, 2009.

8. Carano, R.A.D., and Filvaroff, E.H. Angiogenesis and bone repair. Drug Discov Today 8, 980, 2003.

9. Gerstenfeld, L.C., and Einhorn, T.A. Developmental aspects of fracture healing and the use of pharmacological agents to alter healing. J Musculoskelet Neuronal Interact 3, 297, 2003.

10. Chen, F.M., Zhang, M., and Wu, Z.F. Toward delivery of multiple growth factors in tissue engineering. Biomaterials 31, 6279, 2010.

11. Carragee, E.J., Hurwitz, E.L., and Weiner, B.K. A critical review of recombinant human bone morphogenetic protein2 trials in spinal surgery: emerging safety concerns and lessons learned. Spine J 11, 471, 2011.

12. Bradley, M., Lazim, A.M., and Eastoe, J. Stimulus-responsive heteroaggregation of colloidal dispersions: reversible systems and composite materials. Polymers 3, 1036, 2011.

13. Wang, H., Leeuwenburgh, S.C.G., Li, Y., and Jansen, J.A. The use of micro- and nanospheres as functional components for bone tissue regeneration. Tissue Eng Part B Rev 18, 24, 2012.

14. Wang, H., Zou, Q., Boerman, O., C,, Nijhuis, A.W., Jansen, J.A., Li, Y., et al. Combined delivery of BMP-2 and bFGF from nanostructured colloidal gelatin gels and its effect on bone regeneration in vivo. I Control Release 166, 172, 2013.

15. Fujimura, K., Bessho, K., Okubo, Y., Kusumoto, K., Segami, N., and Iizuka, T. The effect of fibroblast growth factor-2 on the osteoinductive activity of recombinant human bone morphogenetic protein-2 in rat muscle. Arch Oral Biol 47, 577, 2002.

16. Springer, I.N.G., Niehoff, P., Açil, Y., Marget, M., Lange, A., Warnke, P.H., Pielenz, H., Roldán, J.C., and Wiltfang, J. BMP-2 and bFGF in an irradiated bone model. I Craniomaxillofacial Surg 36, 210, 2008.

17. Wang, H., Hansen, M.B., Lowik, D.W., van Hest, J.C., Li, Y., Jansen, J.A., et al. Oppositely charged gelatin nanospheres as building blocks for injectable and biodegradable gels. Adv Mater 23, H119, 2011.

18. Takemoto, M., Fujibayashi, S., Neo, M., Suzuki, J., Matsushita, T., Kokubo, T., et al. Osteoinductive porous titanium implants: effect of sodium removal by dilute $\mathrm{HCl}$ treatment. Biomaterials 27, 2682, 2006.

19. Unger, S., Blauth, M., and Schmoelz, W. Effects of three different preservation methods on the mechanical properties of human and bovine cortical bone. Bone 47, 1048, 2010.

20. Murr, L.E., Gaytan, S.M., Medina, F., Lopez, H., Martinez, E., Machado, B.I., et al. Next-generation biomedical implants using additive manufacturing of complex, cellular and functional mesh arrays. Philos Transact A Math Phys Eng Sci 368, 1999.

21. Marsell, R., and Einhorn, T.A. The biology of fracture healing. Injury 42, 551, 2011.

22. Zara, J.N., Siu, R.K., Zhang, X., Shen, J., Ngo, R., Lee, M., et al. High doses of bone morphogenetic protein 2 induce 
structurally abnormal bone and inflammation in vivo. Tissue Eng Part A 17, 1389.

23. Angle, S.R., Sena, K., Sumner, D.R., Virkus, W.W., and Virdi, A.S. Healing of rat femoral segmental defect with bone morphogenetic protein-2: a dose response study. I Musculoskelet Neuronal Interact 12, 28, 2012.

24. Zellin, G., and Linde, A. Effects of recombinant human fibroblast growth factor-2 on osteogenic cell populations during orthopic osteogenesis in vivo. Bone 26, 161, 2000.

25. Guillame-Gentil, O., Semenov, O., Roca, A.S., Groth, T., Zahn, R., Vörös, J., and Zenobi-Wong, M. Engineering the Extracellular Environment: Strategies for Building 2D and 3D Cellular Structures. Adv Mater 22, 5443, 2010.

26. Luong, L.N., Ramaswamy, J., and Kohn, D.H. Effects of osteogenic growth factors on bone marrow stromal cell differentiation in a mineral-based delivery system. Biomaterials 33, 283, 2012.

27. Tabata, Y., and Ikada, Y. Vascularization effect of basic fibroblast growth factor released from gelatin hydrogels with different biodegradabilities. Biomaterials 20, 2169, 1999.

28. Mayahara, H., Ito, T., Nagai, H., Miyajima, H., Tsukuda, R., Taketomi, S., et al. In vivo stimulation of endosteal bone formation by basic fibroblast growth factor in rats. Growth Factors 9, 73, 1993.

29. Nakamura, Y., Tensho, K., Nakaya, H., Nawata, M., Okabe, T., and Wakitani, S. Low dose fibroblast growth factor-2 (FGF-2) enhances bone morphogenetic protein-2 (BMP-2)induced ectopic bone formation in mice. Bone 36, 399, 2005.
30. Takita, H., Tsuruga, E., Ono, I., and Kuboki, Y. Enhancement by bFGF of osteogenesis induced by rhBMP-2 in rats. Eur J Oral Sci 105, 588, 1997.

31. Maegawa, N., Kawamura, K., Hirose, M., Yajima, H., Takakura, Y., and Ohgushi, H. Enhancement of osteoblastic differentiation of mesenchymal stromal cells cultured by selective combination of bone morphogenetic protein-2 (BMP-2) and fibroblast growth factor-2 (FGF-2). J Tissue Eng Regen Med 1, 306, 2007.

32. Tanaka, E., Ishino, Y., Sasaki, A., Hasegawa, T., Watanabe, M., Dalla-Bona, D., Yamano, E., van Eijden, T., and Tanne, K. Fibroblast Growth Factor-2 Augments Recombinant Human Bone Morphogenetic Protein-2-Induced Osteoinductive Activity. Ann Biomed Eng 34, 717, 2006.

Address correspondence to: Johan van der Stok, MD

Orthopaedic Research Laboratory Department of Orthopaedics Erasmus University Rotterdam Medical Centre Room Ee1614, PO Box 2040 Rotterdam 3000CA The Netherlands

E-mail: j.vanderstok@erasmusmc.nl

Received: March 21, 2013

Accepted: June 20, 2013

Online Publication Date: August 15, 2013 
This article has been cited by:

1. Nazli Sarkalkan, Harrie Weinans, Amir A. Zadpoor. 2013. Statistical shape and appearance models of bones. Bone . [CrossRef]

2. Huanan Wang, Matilde Bongio, Kambiz Farbod, Arnold W.G. Nijhuis, Jeroen van den Beucken, Otto C. Boerman, Jan C.M. van Hest, Yubao Li, John A. Jansen, Sander C.G. Leeuwenburgh. 2013. Development of injectable organic/inorganic colloidal composite gels made of self-assembling gelatin nanospheres and calcium phosphate nanocrystals. Acta Biomaterialia . [CrossRef] 